\title{
Article \\ Psychological Distress among Bangladeshi Dental Students during the COVID-19 Pandemic
}

\author{
Farah Sabrina ${ }^{1}$, Mohammad Tawfique Hossain Chowdhury ${ }^{2}$, Sujan Kanti Nath ${ }^{3}$, Ashik Abdullah Imon ${ }^{4}$, S.M. \\ Abdul Quader ${ }^{5}$, Md. Shahed Jahan ${ }^{6}$, Ashek Elahi Noor ${ }^{7}$, Clopa Pina Podder, Unisha Gainju' ${ }^{9}$, Rina Niroula ${ }^{10}$, \\ Muhammad Aziz Rahman ${ }^{11-13^{*}}$
}

1 Department. of Oral \& Maxillofacial Surgery, Update Dental College, Dhaka, Bangladesh; sabrinafarahbds40@gmail.com

2 Department of Dental Public Health, Sapporo Dental College, Dhaka, Bangladesh; drtawfique@hotmail.com

3 Department. of Dental Public Health Sapporo Dental College, Dhaka, Bangladesh; knsujan@yahoo.com

4 Department. of Oral \& Maxillofacial Surgery, Update Dental College, Dhaka, Bangladesh; imonomfs@gmail.com

5 Department of Conservative Dentistry \& Endodontics, Update Dental College, Dhaka, Bangladesh; smilezonedental@yahoo.com

6 Department of Dental Public Health, Update Dental College, Dhaka, Bangladesh; shahed.jahan5@gmail.com

7 Department. of Dental Public Health Sapporo Dental College, Dhaka, Bangladesh; rajet.elahi@gmail.com

8 Department of Dental Public Health, Sapporo Dental College, Dhaka, Bangladesh; drcppodder@gmail.com

9 Update Dental College, Dhaka, Bangladesh; unesa97@gmail.com

10 Update Dental College, Dhaka, Bangladesh; niroularina@gmail.com

11 School of Health, Federation University Australia, Berwick, Victoria, Australia, ma.rahman@federation.edu.au

12 Department of Non-communicable Diseases, Bangladesh University of Health Sciences (BUHS), Dhaka, Bangladesh

13 Faculty of Public Health, Universitas Airlangga, Surabaya, Indonesia

* Correspondence: (MAR) ma.rahman@federation.edu.au , Tel.: + 6134313 7908; (MTHC) drtawfique@hotmail.com , Tel: +88 01720245861

\begin{abstract}
:
Background: Psychological sufferings are observed among dental students during their academic years, which had been intensified during the COVID-19 pandemic. Objectives: This study assessed the levels and identified factors associated with psychological distress, fear and coping experienced by dental undergraduate students in Bangladesh. Methods: A cross sectional online survey was conducted during October-November, 2021. The Kessler Psychological Distress Scale (K-10), Fear of COVID-19 Scale (FCV-19S) and Brief Resilient Coping Scale (BRCS) were used in order to assess psychological distress, fear and coping strategies respectively. Results: A total of 327 students participated; the majority (72\%) were $19-23$ years old and females (75\%). One in five participants were infected with COVID-19 and 15\% reported contact with COVID-19 cases. Negative financial impact (AOR 3.72, 95\%CIs 1.28-10.8), recent or past COVID-19 infection, contact with COVID-19 cases were associated with higher levels of psychological distress; but being a $3^{\text {rd }}$ year student $(0.14,0.04-0.55)$ and being satisfied about current social life $(0.11,0.03-0.33)$ were associated with lower levels of psychological distress. Being a $3^{\text {rd }}$ year $(0.17,0.08-0.39)$ and a $4^{\text {th }}$ year student $(0.29,0.12-0.71)$ were associated with lower levels of fear. Health care service use and feeling positive about life were associated with medium to high resilience coping. Conclusions: This study identified dental students in Bangladesh who were at higher risk of psychological distress, fear and coping during the ongoing pandemic. Development of mental health support system within dental institution should be considered in addition to the academic and clinical teaching.
\end{abstract}

Keywords: Psychological distress; Fear; Coping; COVID-19; Bangladesh; Dental; Mental health 


\section{Introduction}

The ongoing coronavirus disease (COVID-19) pandemic has been linked to more than 140 million cases worldwide, with approximately 3 million deaths [1]. The pandemic has caused the most cases and deaths in the United States of America, followed by India, Brazil, France, the Russian Federation, the United Kingdom, Turkey, Italy, and Spain. The first three cases of COVID-19 were identified in Bangladesh on March 8, 2020. Till 29th Nov, 2021, Bangladesh has reported 1,575,579 confirmed COVID-19 cases and 27,975 deaths [2]. In response to the pandemic crisis, the Government of the People's Republic of Bangladesh has designed a Multisectoral Action Plan. Lockdown in major cities, social distancing, closure of schools and universities, working from home arrangements where possible, widespread public awareness campaigns for handwashing practices, use of masks in public places, imposing regulations on international travel from hotspots, managing quarantine centers and nationwide testing facilities were few of the initiatives taken by the Government to mitigate the impact of the pandemic. In addition, guidelines for COVID-19 clinical management were developed, public and private hospitals were designated for treating positive cases, isolation units were established in various hospitals, and regular public reporting was initiated based on the surveillance of COVID-19 cases and deaths $[3,4]$.

The pandemic has impacted global communities in different ways. Besides the impact on physical health, it also triggered a slew of psychological issues, including panic disorder, anxiety, and sadness, in both COVID-19 patients and healthy people [8]. Due to the contagious nature of COVID-19, concerns such as spatial segregation, lockdown, travel limitations, and isolation, as well as social and economic ramifications, resulting in despair, anxiety, fear, panic, stress, suicidal thoughts, post-traumatic stress disorder and other mental health issues [9]. A recent study examining factors associated with psychological distress, fear of COVID-19, and coping across diverse community members in 17 countries showed that doctors had greater levels of psychological distress but lower levels of fear of COVID-19, whereas nurses had higher levels of resilient coping. Females and individuals with pre-existing mental health issues were identified as the most vulnerable groups of people having COVID-related psychological impact [7]. Bangladeshi individuals also experienced a great deal of psychological discomfort and terror, according to a recent study [5]. People with pre-existing mental health problems, females, frontline workers or essential service worker, being an ever smoker, providing care to a known or suspected case of COVID-19, having an overseas travel history, being in quarantine, having positive test results for COVID-19, and having higher levels of fear of COVID-19 were associated with higher psychological distress [5].

The pandemic has posed a challenge to healthcare workers including dental clinicians around the world, prompting a variety of responses. Medical and Dental schooling are widely seen as demanding environments, with students experiencing higher levels of stress, anxiety, and depression than classmates studying other subjects [10]. Obtaining a Bachelor's degree in Dentistry is a time-consuming process that demands extensive study and expertise of the discipline. In Bangladesh, the program is of five years duration, with the last two years dedicated to clinical training and a yearlong internship following graduation. An undergraduate Dentistry student needs to demonstrate theoretical knowledge, practical experience, and interpersonal skills, all of which are assessed at the end of each academic year via oral, written, and practical assessments. These ongoing academic responsibilities, as well as non-academic stress such as coordinating with faculty and administrative formalities, are often overwhelming for students [11,12]. Most dental treatments, particularly those involving the use of a dental hand piece, produce aerosols. During the pandemic, that practice potentially increased the risk of spreading infection at the dentist practice. It has been demonstrated that the virus in the aerosol may 
survive for more than 3 hours, with surface stability over 72 hours [13]. Development of that scientific evidence generated anxiety and stress amongst the students doing their clinical placement. Many countries have postponed elective dental procedures, and a few countries have even closed dental schools, clinics and teaching hospitals [14,15]. For months, not only dental clinics, but also dental schools, dental teaching hospitals and universities were closed in many countries like USA, Canada, Japan, China, India etc. [16-18]. In addition, all academic dental institutions, dental clinics, in Bangladesh were temporarily closed during the pandemic. Only emergency dental treatments were provided.

Following the necessity for social distancing due to the COVID-19 pandemic, physical presence at schools, colleges, and universities all around the world were restricted and transitioned to virtual learning environment. There were similar arrangements for Dental schools all over the world including Bangladesh. Such a new way of learning allowed the academics and students to gain more personalized educational experience. In addition, for preclinical simulation exercises, certain teaching institutes adopted the social distancing methods in their dental laboratories following the strict COVID-19 guidelines (for example: students were divided into small subgroups in their clinical class, wearing masks, face shields, using hand sanitizers) [19-21]. However, evidence showed that Dental and Medical students suffered from psychological anguish due to the change in learning environment during their academic and professional years [22]. Dental students reported a number of mental health issues including depression, anxiety, obsessive-compulsive disorders and interpersonal sensitivity in their academic years during the current pandemic [23-26].

Studies focusing on the impact of COVID-19 on Bangladeshi students, specifically, who were pursuing studies on health sciences including dentistry were very limited. However, it was important to assess their psychological impact not only due to the pandemic, but also due to the changed learning environment and clinical training. Therefore, we aimed to assess psychological distress, fear of COVID-19 and coping amongst Bangladeshi dental students and identify factors associated with those issues.

\section{Materials and Methods}

\subsection{Study design}

A cross-sectional study was conducted between October to November 2021 where students of two different private Dental colleges participated via online platform.

\subsection{Study sites}

Two large private Dental Colleges in the capital city of Bangladesh were selected as the study sites. Both sites had both teaching and clinical training facilities besides outdoor services. The first site had 444 students with 130 patients used to attend the hospital daily; the second site had 318 students with daily visit of 60 patients.

\subsection{Study population}

Current students of those two study sites from $1^{\text {st }}$ to $4^{\text {th }}$ academic years were eligible for this study. Because of the inaccuracy of the responses, any study participant who took more than 1 minute to complete the questionnaire was omitted from the analyses.

\subsection{Sampling}


The Snowball sampling technique was used for collecting data. Once a participant filled up the online questionnaire, he/she was requested to forward the survey link to his/her personal/professional networks. Sample size was calculated using Open Epi. Considering total students of 759 from two study sites, expected frequency of psychological distress as $70 \%$ based on the previous study in Bangladesh [5], 95\% confidence intervals and $80 \%$ power, the estimated minimum sample size was 227.

\subsection{Study questionnaire}

A structured survey questionnaire was used for data collection in this study and was adopted from a global study led by the lead author of this study (MAR) [7]. Google forms were used to create the survey. The first section of the study questionnaire collected sociodemographic data as well as information on physical symptoms of COVID-19, history of contacts with COVID-19 cases, self-reported comorbidities, behavioral risk factors, health service utilization in the last four weeks including type of service providers and access to mental health resources. Psychological impact was assessed by the Kessler Psychological Distress Scale (K-10) [27], fear was assessed by the Fear of COVID-19 scale (FCV-19S) [28] and coping strategies were assessed by the Brief Resilient Coping Scale (BRCS) [29]. The details of each tool including the items were discussed in our earlier published studies [6]. A pretest of the questionnaire was performed on a selective group of participants and the necessary modification was done before the data collection.

\subsection{Data collection}

The online link of the survey was emailed to all the students at both sites inviting them to participate. The volunteer nature of the study was highlighted. Data were collected during October- November, 2021. On the first screen, the plain language information statement (PLIS) and consent form were displayed. Only those who provided consent, could proceed to the following screens. The following screens displayed the entire study questionnaire, which consisted of 46 items.

\subsection{Data analyses}

Data were analyzed using SPSS v.25. At first, descriptive analyses were conducted. Categorical variables were reported as proportions and continuous variables were reported as mean $( \pm S D)$. In that way, levels of psychological distress, fear of COVID-19 and coping were reported. Then inferential analyses were conducted to identify the factors associated with those outcomes. At first, chi-squared tests were conducted to determine existence of association and $\mathrm{p}<0.05$ was considered significant. Then univariate logistic regression was conducted to determine the strength of association; odds ratios (ORs) and 95\% confidence intervals (CIs) were reported. Finally, multivariate logistic regression was conducted to control potential confounders; adjusted ORs (AORs) and 95\% CIs were reported.

\subsection{Ethics}

The study protocol was reviewed and approved by the Human Research Ethics Committee at one of the study sites (ref no: SDC/C7/2021/829). The survey was completely voluntary in nature and it was clarified in the PLIS, so that participants got the opportunity to have informed decision to participate in the study. No identifying information including any personal sensitive information were collected. Responses were anonymous and nonidentifiable data were handled only by the study investigators.

\section{Results}


A total of 327 Bangladeshi dental undergraduate students participated in the study. The majority $(71.6 \%)$ of the students belonged to the age group of 19-23 years and were females $(74.9 \%)$. The mean age $( \pm \mathrm{SD})$ was $22.5( \pm 1.7)$ years with the majority $(62.4 \%)$ from clinical years (3rd and 4 th year). Almost all of the participants $(95.1 \%)$ reported financial dependence on their families and more than half (58.1\%) reported that the pandemic negatively impacted on their financial situation; yet most of them $(81.9 \%)$ were satisfied with their current social life.

About one in five participants (19.6\%) were infected with COVID-19, although recent infection was reported as only $2.4 \%$. More than one in ten participants $(15 \%)$ reported that they were involved in direct or indirect care of their family or friends who were infected with COVID-19. Other characteristics of the study population are reported in Table-1.

Table 1. Characteristics of study population

\begin{tabular}{|c|c|}
\hline Characteristics & Total, n(\%) \\
\hline Total study participants & 327 \\
\hline Age (in years) & 327 \\
\hline Mean $( \pm$ SD) & $22.5(1.7)$ \\
\hline Age groups & 327 \\
\hline $19-23$ years & 234(71.6) \\
\hline $24-28$ years & $93(28.4)$ \\
\hline Gender & 327 \\
\hline Male & $82(25.1)$ \\
\hline Female & $245(74.9)$ \\
\hline Marital status & 327 \\
\hline Married & $48(14.7)$ \\
\hline Unmarried & $278(85.0)$ \\
\hline Divorced & $1(.3)$ \\
\hline Family types & 327 \\
\hline Nuclear family & $266(81.3)$ \\
\hline Joint family & $61(18.7)$ \\
\hline Living status & 327 \\
\hline Live without family members & $11(3.4)$ \\
\hline Live with family members & $151(46.2)$ \\
\hline Live in own house & $7(2.1)$ \\
\hline Live in shared house & $16(4.9)$ \\
\hline Live in hostel & $142(43.4)$ \\
\hline Perceived safety of living place in relation to COVID-19 & 327 \\
\hline Unsafe & $50(15.3)$ \\
\hline Safe & 277(84.7) \\
\hline Year of Dental education & 327 \\
\hline 1st year & $68(20.8)$ \\
\hline 2nd year & $55(16.8)$ \\
\hline 3rd year & $98(30.0)$ \\
\hline 4 th year & $106(32.4)$ \\
\hline
\end{tabular}


Financial contribution to family

Fully dependent on family

311(95.1)

Part of earning goes to family

16(4.9)

COVID-19 impacted financial situation

327

No impact

82(25.1)

Yes, impacted positively

$54(16.5)$

Yes, impacted negatively

190(58.1)

Perceived current social life

327

Dissatisfied

$59(18.0)$

Satisfied

268(81.9)

Co-morbidities

327

No

284(86.9)

Diabetes

3(0.9)

Hypertension

$7(2.1)$

Tuberculosis

$1(0.3)$

Chronic kidney disease

Lung disease

Carcinoma

Others

Smoking

327

Never smoker

311(95.1)

Ever smoker (Daily/Non-daily/Ex)

16(4.9)

327

Infected with COVID-19

227(69.4)

No

64(19.6)

Yes

$36(11)$

Don't know

Number of times infected with COVID-19

$1.14( \pm 0.393)$

Mean $( \pm S D)$

Infected with COVID-19 in the last 14 days

$319(97.6)$

No

$8(2.4)$

Yes

No

259(79.2)

Yes

$46(14.1)$

May be

22(6.7)

327

No

$278(85.0)$

Unsure

$31(9.5)$

Yes

18(5.5)

Activities during lockdown (multiple responses)

Reading books

Watching movies 
Doing household chores

Listening to music

Engaging in social media

Cooking

Studying

Gardening

Others

Experience related to the use of social media

Do not use

Does not affect

Find it irritating

Feel positive about life

Never

Quite often

Always positive

Faced difficulties in adopting distance learning

No

Yes

Healthcare service use to overcome COVID-19 related stress in the last 6

months

No

Type of healthcare service used to overcome COVID-19 related stress in the last 6 months

Consulted a GP

Consulted a Psychologist

Consulted a Psychiatrist

Others

Though more than half of the participants reported low levels of fear of COVID-19 $(53.8 \%)$, most of them $\mathrm{s}$ experienced moderate to high level of psychological distress $(84.2 \%)$ with more than half $(60.2 \%)$ being low resilient copers.

\subsection{Psychological Distress}

Univariate analyses showed that perceived safety in living places, being 3rd year clinical dental students, negative impact of COVID-19 over financial situation, perceived satisfaction with current social life, irritating experience related to use of social media and feeling positive about life were significantly associated with moderate to very high levels of psychological distress compared to their counterparts. After adjustment of potential confounders, those who were at the 3rd year of their academic year (AOR 0.14, 95\% CIs 0.04$0.55, p=0.005$ ) and who reported satisfaction with current social life (AOR 0.11,95\% CIs $0.03-0.33, p<0.001)$ were less likely to report moderate to very high levels of psychological distress. On the other hand, those who reported negative impact of COVID-19 over financial situation (AOR 3.72, 95\% CIs 1.28-10.8, $\mathrm{p}=0.015$ ), , who were infected with COVID-19 both recently (and in the past, who were unsure of the contact with COVID-19 cases were more likely to report moderate to very high levels of psychological distress. (Table-5) 
Table 2. Level of psychological distress among the study participants

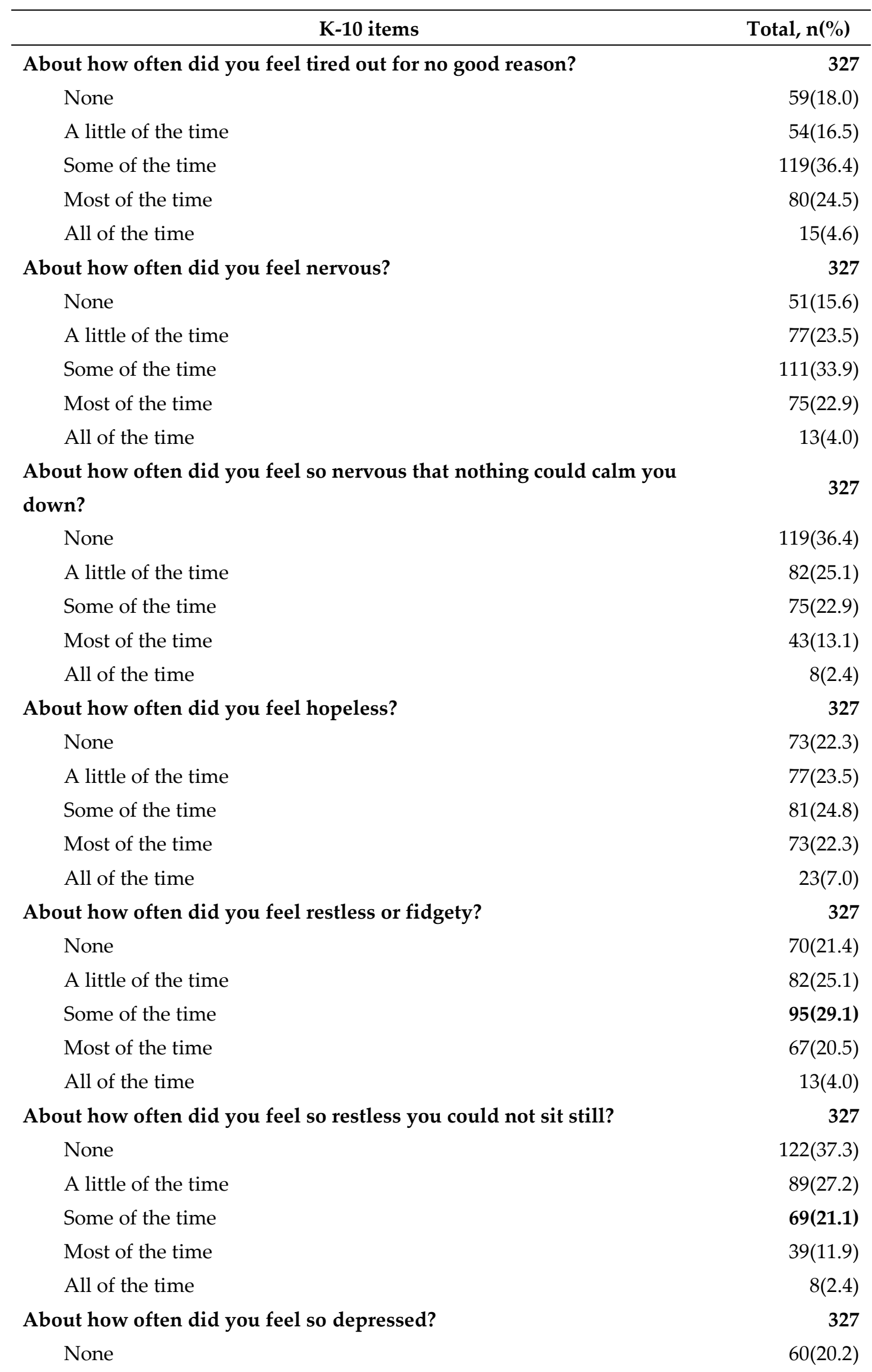


A little of the time

$71(21.7)$

Some of the time

88(26.9)

Most of the time

$75(22.9)$

All of the time

$27(8.3)$

327

About how often did you feel that everything was an effort?

60(18.3)

None

$53(16.2)$

A little of the time

130(39.8)

Some of the time

61(18.7)

Most of the time

23(7.0)

All of the time

None

A little of the time

$82(25.1)$

Some of the time

89(27.2)

Most of the time

61(18.7)

All of the time

None

A little of the time

Some of the time

Most of the time

All of the time

K10 score (total)

Mean $( \pm$ SD)

Level of psychological distress (K10 categories)

Low (score 10-15)

Moderate (score 16-21)

High (score 22-29)

Very high (score 30-50)

\subsection{Levels of Fear}

Univariate analyses showed that being female and those who were living in joint families were more likely to report high levels of fear of COVID-19. On the contrary, being a student of 2 nd, 3rd and 4th year, being a smoker and those who were medium to high resilient copers were more likely to report low levels of fear of COVID-19. After adjustment of the potential confounders, it was found that those who were at the 3rd year (AOR 0.17, 95\% CIs $0.08-0.39, \mathrm{p}<0.001$ ) and 4 th year (AOR $0.29,95 \%$ CIs $0.12-0.71, \mathrm{p}=0.006$ ) clinical students had low levels of fear of COVID-19.(Table-6)

Table 3. Level of fear of COVID-19 among the study participants 
Strongly disagree

Disagree

65(19.9)

Neither agree nor disagree

92(28.1)

Agree

127(38.8)

Strongly agree

16(4.9)

327

Strongly disagree

Disagree

71(21.7)

Neither agree nor disagree

77(23.5)

Agree

147(45.0)

Strongly agree

$17(5.2)$

327

Strongly disagree

39(11.9)

Disagree

128(39.1)]

Neither agree nor disagree

72(22.0)

Agree

83(25.4)

Strongly agree

327

Strongly disagree

Disagree

87(26.6)

Neither agree nor disagree

$70(21.4)$

Agree

118(36.1)

Strongly agree 22(6.7)

When watching news and stories about COVID-19 on social media, I become nervous or anxious

Strongly disagree

Disagree

Neither agree nor disagree

70(21.4)

Agree

168(51.4)

Strongly agree

I cannot sleep because I'm worrying about getting COVID-19

Strongly disagree

55(16.8)

Disagree

153(46.8)

Neither agree nor disagree

$79(24.2)$

Agree

37(11.3)

Strongly agree

3(.9)

My heart races or palpitates when I think about getting COVID-19

Strongly disagree

Disagree

Neither agree nor disagree

Agree

87(26.6)

Strongly agree 
FCV-19S score (total)

Mean $( \pm S D)$

$20.4(5.4)$

Level of fear of COVID-19 (FCV-19S categories)

Low (score 7-21)

$176(53.8)$

High (score 22-35)

151(46.2)

\subsection{Coping strategies}

Univariate analyses demonstrated that those who were living in hostel, and those who had high level of COVID-related fear were more likely to be low resilient copers. On the other hand, those who were quite often positive about life and those who used health care service to overcome COVID-19 related stress were more likely to be medium to high resilient copers. However, after adjustment of the potential confounders, it was revealed that those who were females (AOR 0.47, 95\% CIs 0.24-0.93, $\mathrm{p}=0.030$ ), living in hostel (AOR 0.51, 95\% CIs $0.29-0.89, \mathrm{P}=0.018$ ) and who were quite often positive about life (AOR 3.67, 95\% CIs 1.17-11.5, $\mathrm{P}=0.026$ ) were more likely to be low resilient copers; those who were quite often positive about life (AOR 3.67, 95\% CIs 1.17-11.5, $\mathrm{p}=0.026$ ) and those who used health care service to overcome COVID-19 related stress (AOR 2.19, 95\% CIs 1.15-4.17, $\mathrm{p}=0.017$ ) were more likely to be medium to high resilient copers. (Table-7)

Table 4. Coping during the COVID-19 pandemic among the study participants

\begin{tabular}{lr}
\hline \multicolumn{1}{c}{ BRCS items } & $\begin{array}{r}\text { Total, } \\
\mathbf{n}(\%)\end{array}$ \\
\hline I look for creative ways to alter difficult situations & 327 \\
Does not describe me at all & $16(4.9)$ \\
Does not describe me & $21(6.4)$ \\
Neutral & $186(56.9)$ \\
Describes me & $82(25.1)$ \\
Describes me very well & $22(6.7)$ \\
Regardless of what happens to me, I believe I can control my reaction to it & 327 \\
Does not describe me at all & $16(4.9)$ \\
Does not describe me & $27(8.3)$ \\
Neutral & $180(55.0)$ \\
Describes me & $79(24.2)$ \\
Describes me very well & $25(7.6)$ \\
I believe I can grow in positive ways by dealing with difficult situations & 327 \\
Does not describe me at all & $9(2.8)$ \\
Does not describe me & $19(5.8)$ \\
Neutral & $154(47.1)$ \\
Describes me & $112(34.3)$ \\
Describes me very well & $33(10.1)$ \\
I actively look for ways to replace the losses I encounter in life & 327 \\
Does not describe me at all & $13(4.0)$ \\
Does not describe me & $24(7.3)$ \\
Neutral & $190(58.1)$
\end{tabular}


Describes me

Describes me very well

20(6.1)

BRCS score (total)

Mean $( \pm S D)$

$13.1(2.6)$

Level of coping (BRCS categories)

327

Low resilient copers (score 4-13)

197(60.2)

Medium resilient copers (score 14-16)

102(3.2)

High resilient copers (score 17-20)

28(8.6) 
Table 5. Predictors for high psychological distress among the study population (based on K10 score)

\begin{tabular}{|c|c|c|c|c|c|c|c|c|c|c|}
\hline Characteristics & \multicolumn{2}{|c|}{$\begin{array}{c}\text { Low (score } \\
10-15)\end{array}$} & \multicolumn{2}{|c|}{$\begin{array}{l}\text { Moderate to } \\
\text { Very High } \\
\text { (score 16-50) }\end{array}$} & \multicolumn{3}{|c|}{ Unadjusted analyses } & \multicolumn{3}{|c|}{ Adjusted analyses } \\
\hline Age groups & 52 & & 275 & & \multirow{2}{*}{\multicolumn{3}{|c|}{1}} & \multirow{2}{*}{\multicolumn{3}{|c|}{1}} \\
\hline $19-23$ years & 38 & 16.2 & 196 & 83.8 & & & & & & \\
\hline $24-28$ years & 14 & 15.1 & 79 & 84.9 & 0.791 & 1.09 & $0.56-2.13$ & 0.188 & 0.43 & $0.12-1.51$ \\
\hline Male & 18 & 22 & 64 & 78 & \multicolumn{3}{|c|}{1} & \multicolumn{3}{|c|}{1} \\
\hline Female & 34 & 13.9 & 211 & 86.1 & 0.086 & 1.75 & $0.92-3.30$ & 0.130 & 2.25 & $0.79-6.45$ \\
\hline Marital status & 52 & & 275 & & & & & & & \\
\hline Married & 8 & 16.7 & 40 & 83.3 & \multicolumn{3}{|c|}{1} & \multicolumn{3}{|c|}{1} \\
\hline Unmarried & 44 & 15.8 & 234 & 84.2 & 0.883 & 1.06 & $0.47-2.43$ & 0.465 & 0.63 & $0.18-2.19$ \\
\hline Family types & 52 & & 275 & & & & & & & \\
\hline Live without family members & 1 & 9.1 & 10 & 90.9 & \multicolumn{3}{|c|}{1} & \multicolumn{3}{|c|}{1} \\
\hline Live with family members & 23 & 15.2 & 128 & 84.8 & 0.585 & 1.80 & $0.22-14.7$ & 0.299 & 4.26 & $0.28-65.4$ \\
\hline Live in own house & 2 & 28.6 & 5 & 71.4 & 0.356 & 0.45 & $0.08-2.46$ & 0.412 & 0.38 & $0.04-3.88$ \\
\hline Live in shared house & 4 & 25 & 12 & 75 & 0.319 & 0.54 & $0.16-1.82$ & 0.609 & 1.58 & $0.28-9.01$ \\
\hline Live in hostel & 22 & 15.5 & 120 & 84.5 & 0.951 & 0.98 & $0.52-1.85$ & 0.802 & 1.14 & $0.41-3.15$ \\
\hline Perceived safety of living place in relation to COVID-19 & 52 & & 275 & & & & & & & \\
\hline Unsafe & 3 & 6 & 47 & 94 & \multicolumn{3}{|c|}{1} & \multicolumn{3}{|c|}{1} \\
\hline Safe & 49 & 17.7 & 228 & 82.3 & 0.049 & 0.30 & $0.09-0.99$ & 0.970 & 1.03 & $0.17-6.21$ \\
\hline Year of Dental education & 52 & & 275 & & & & & & & \\
\hline 1 st year & 7 & 10.3 & 61 & 89.7 & \multicolumn{3}{|c|}{1} & \multicolumn{3}{|c|}{1} \\
\hline
\end{tabular}




$$
\begin{aligned}
& \text { 2nd year } \\
& \text { 3rd year } \\
& \text { 4th year }
\end{aligned}
$$

Financial contribution to family

Fully dependent on family

Part of earning goes to family

COVID-19 impacted financial situation

$$
\begin{aligned}
& \text { No impact } \\
& \text { Yes, impacted positively } \\
& \text { Yes, impacted negatively }
\end{aligned}
$$

Perceived current social life

$$
\begin{aligned}
& \text { Dissatisfied } \\
& \text { Satisfied }
\end{aligned}
$$

\section{Smoking}

Never smoker

Ever smoker (Daily/Non-daily/Ex)

Infected with COVID-19

No

$$
\text { Yes }
$$

\begin{tabular}{|c|c|c|c|c|c|c|c|c|c|}
\hline 5 & 9.1 & 50 & 90.9 & 0.823 & 1.15 & $0.34-3.84$ & 0.718 & 0.74 & $0.15-3.74$ \\
\hline 29 & 29.6 & 69 & 70.4 & 0.004 & 0.27 & $0.11-0.67$ & 0.005 & 0.14 & $0.04-0.55$ \\
\hline 11 & 10.4 & 95 & 89.6 & 0.986 & 0.99 & $0.36-2.70$ & 0.858 & 0.87 & $0.19-4.07$ \\
\hline 52 & & 275 & & & & & & & \\
\hline 49 & 15.8 & 262 & 84.2 & & 1 & & & 1 & \\
\hline 3 & 18.8 & 13 & 81.3 & 0.750 & 0.81 & $0.22-2.95$ & 0.953 & 1.07 & $0.13-8.62$ \\
\hline 52 & & 275 & & & & & & & \\
\hline 26 & 31.7 & 56 & 68.3 & & 1 & & & 1 & \\
\hline 9 & 16.7 & 45 & 83.3 & 0.053 & 2.32 & $0.99-5.45$ & 0.288 & 2.01 & $0.56-7.24$ \\
\hline 17 & 8.9 & 173 & 91.1 & 0.000 & 4.72 & $2.39-9.34$ & 0.015 & 3.72 & $1.28-10.8$ \\
\hline 52 & & 275 & & & & & & & \\
\hline 10 & 5.6 & 170 & 94.4 & & 1 & & & 1 & \\
\hline 42 & 28.6 & 105 & 71.4 & 0.000 & 0.15 & $0.07-0.31$ & 0.000 & 0.11 & $0.03-0.33$ \\
\hline 52 & & 275 & & & & & & & \\
\hline 51 & 16.4 & 260 & 83.6 & & 1 & & & 1 & \\
\hline 1 & 6.3 & 15 & 93.8 & 0.301 & 2.94 & $0.38-22.8$ & 0.232 & 6.22 & $0.31-125$ \\
\hline 52 & & 275 & & & & & & & \\
\hline 40 & 17.6 & 187 & 82.4 & & 1 & & & 1 & \\
\hline 8 & 12.5 & 56 & 87.5 & 0.215 & 1.36 & $0.84-2.22$ & 0.030 & 2.52 & $1.10-5.78$ \\
\hline 52 & & 275 & & & & & & & \\
\hline 51 & 16 & 268 & 84 & & 1 & & & 1 & \\
\hline 1 & 12.5 & 7 & 87.5 & 0.791 & 1.33 & $0.16-11.1$ & 0.022 & 62.7 & $1.81-2175$ \\
\hline 52 & & 275 & & & & & & & \\
\hline 44 & 17 & 215 & 83 & & 1 & & & 1 & \\
\hline 6 & 13 & 40 & 87 & 0.384 & 1.23 & $0.78-1.93$ & 0.104 & 0.48 & $0.20-1.17$ \\
\hline 52 & & 275 & & & & & & & \\
\hline 45 & 16.2 & 233 & 83.8 & & 1 & & & 1 & \\
\hline 3 & 9.7 & 28 & 90.3 & 0.349 & 1.80 & $0.53-6.18$ & 0.030 & 8.38 & $1.23-56.9$ \\
\hline
\end{tabular}

Infected with COVID-19 in the last 14 days

$$
\begin{aligned}
& \text { No } \\
& \text { Yes }
\end{aligned}
$$

Experienced symptoms of COVID-19 in the last 14 days

$$
\text { No }
$$$$
\text { Yes }
$$

Contact (indirect/direct) with COVID-19 cases

$$
\text { No }
$$

Unsure 
Yes

Experience related to the use of social media

Do not use

Does not affect

Find it irritating

Feel positive about life

Never

Quite often

Always positive

Faced difficulties in adopting distance learning

No

Yes

Level of fear of COVID-19 (FCV-19S categories)

Low (score 7-21)

High (score 22-35)

Level of coping (BRCS categories)

Low resilient copers (score 4-13)

Medium to high resilient copers (score 14-20)

Healthcare service use to overcome COVID-19 related stress in the last 6 months

No

Yes

Type of healthcare service used to overcome COVID-19 related stress in the last 6 months

Consulted a GP

Consulted a Psychologist

Consulted a Psychiatrist

Others

\begin{tabular}{|c|c|c|c|c|c|c|c|c|c|}
\hline 4 & 22.2 & 14 & 77.8 & 0.507 & 0.68 & $0.21-2.15$ & 0.279 & 0.32 & $0.04-2.51$ \\
\hline 52 & & 275 & & & & & & & \\
\hline 4 & 36.4 & 7 & 63.6 & & 1 & & & 1 & \\
\hline 32 & 29.4 & 77 & 70.6 & 0.630 & 1.38 & $0.38-5.02$ & 0.870 & 1.16 & $0.19-7.16$ \\
\hline 16 & 7.7 & 191 & 92.3 & 0.005 & 6.82 & $1.80-25.8$ & 0.158 & 3.88 & $0.59-25.5$ \\
\hline 52 & & 275 & & & & & & & \\
\hline 1 & 4 & 24 & 96 & & 1 & & & 1 & \\
\hline 3 & 2 & 147 & 98 & 0.544 & 2.040 & $0.20-20.4$ & 0.174 & 7.16 & $0.42-122$ \\
\hline 48 & 31.6 & 104 & 68.4 & 0.020 & 0.09 & $0.01-0.69$ & 0.292 & 0.26 & $0.02-3.17$ \\
\hline 52 & & 275 & & & & & & & \\
\hline 12 & 25.5 & 35 & 74.5 & & 1 & & & 1 & \\
\hline 40 & 14.3 & 240 & 85.7 & 0.055 & 2.06 & $0.99-4.30$ & 0.060 & 2.95 & $0.96-9.12$ \\
\hline 52 & & 275 & & & & & & & \\
\hline 26 & 14.8 & 150 & 85.2 & & 1 & & & 1 & \\
\hline 26 & 17.2 & 125 & 82.8 & 0.547 & 0.83 & $0.46-1.51$ & 0.117 & 0.45 & $0.16-1.22$ \\
\hline 52 & & 275 & & & & & & & \\
\hline 34 & 17.3 & 163 & 82.7 & & 1 & & & 1 & \\
\hline 18 & 13.8 & 112 & 86.2 & 0.410 & 1.30 & $0.70-2.41$ & 0.109 & 0.44 & $0.16-1.20$ \\
\hline 52 & & 275 & & & & & & & \\
\hline 39 & 16 & 204 & 84 & & 1 & & & 1 & \\
\hline 13 & 15.5 & 71 & 84.5 & 0.901 & 0.96 & $0.48-1.90$ & 0.267 & 0.52 & $0.16-1.65$ \\
\hline 6 & 17.1 & 29 & 82.9 & 0.928 & 1.06 & $0.32-3.51$ & 0.700 & 1.61 & $0.14-18.3$ \\
\hline 5 & 26.3 & 14 & 73.7 & 0.252 & 0.48 & $0.13-1.69$ & 0.957 & 0.93 & $0.08-11.2$ \\
\hline 1 & 6.3 & 15 & 93.8 & 0.208 & 3.91 & $0.47-32.7$ & 0.299 & 5.00 & $0.24-104$ \\
\hline 1 & 25 & 3 & 75 & 0.690 & 0.62 & $0.06-6.49$ & NA & NA & NA \\
\hline
\end{tabular}


Table 6. Predictors for fear of COVID-19 among the study population (based on FCV-19S score)

\begin{tabular}{|c|c|c|c|c|c|c|c|c|c|c|}
\hline \multirow[t]{2}{*}{ Characteristics } & \multicolumn{2}{|c|}{$\begin{array}{c}\text { Low (score 7- } \\
\text { 21) }\end{array}$} & \multicolumn{2}{|c|}{$\begin{array}{c}\text { High (score 22- } \\
35)\end{array}$} & \multicolumn{3}{|c|}{ Unadjusted analyses } & \multicolumn{3}{|c|}{ Adjusted analyses } \\
\hline & $\mathrm{n}$ & $\%$ & $\mathrm{n}$ & $\%$ & $p$ & ORs & $95 \% \mathrm{Cls}$ & $p$ & AORs & $95 \% \mathrm{Cls}$ \\
\hline Age groups & 176 & & 151 & & \multirow{2}{*}{\multicolumn{3}{|c|}{1}} & \multirow{2}{*}{\multicolumn{3}{|c|}{1}} \\
\hline $19-23$ years & 118 & 50.4 & 116 & 49.6 & & & & & & \\
\hline $24-28$ years & 58 & 62.4 & 35 & 37.6 & 0.052 & 0.61 & $0.38-1.00$ & 0.717 & 0.87 & $0.42-1.81$ \\
\hline Gender & 176 & & 151 & & & & & & & \\
\hline Marital status & 176 & & 151 & & & & & & & \\
\hline Married & 26 & 54.2 & 22 & 45.8 & \multicolumn{3}{|c|}{1} & \multicolumn{3}{|c|}{1} \\
\hline Unmarried & 150 & 54 & 128 & 46 & 0.979 & 1.01 & $0.55-1.86$ & 0.766 & 0.89 & $0.41-1.93$ \\
\hline Family types & 176 & & 151 & & & & & & & \\
\hline Nuclear family & 151 & 56.8 & 115 & 43.2 & \multicolumn{3}{|c|}{1} & \multicolumn{3}{|c|}{1} \\
\hline Live with family members & 78 & 51.7 & 73 & 48.3 & 0.853 & 0.89 & $0.26-3.04$ & 0.605 & 0.67 & $0.14-3.11$ \\
\hline Live in own house & 4 & 57.1 & 3 & 42.9 & 0.777 & 0.80 & $0.17-3.70$ & 0.790 & 0.79 & $0.13-4.61$ \\
\hline Live in shared house & 11 & 68.8 & 5 & 31.3 & 0.200 & 0.49 & $0.16-1.47$ & 0.107 & 0.31 & $0.08-1.29$ \\
\hline Live in hostel & 77 & 54.2 & 65 & 45.8 & 0.660 & 0.90 & $0.57-1.43$ & 0.181 & 0.68 & $0.39-1.20$ \\
\hline Perceived safety of living place in relation to COVID-19 & 176 & & 151 & & & & & & & \\
\hline Unsafe & 25 & 50 & 25 & 50 & \multicolumn{3}{|c|}{1} & \multicolumn{3}{|c|}{1} \\
\hline Safe & 151 & 54.5 & 126 & 45.5 & 0.556 & 0.83 & $0.46-1.52$ & 0.251 & 0.64 & $0.30-1.37$ \\
\hline Year of Dental education & 176 & & 151 & & & & & & & \\
\hline 1st year & 21 & 30.9 & 47 & 69.1 & \multicolumn{3}{|c|}{1} & \multicolumn{3}{|c|}{1} \\
\hline 2nd year & 28 & 50.9 & 27 & 49.1 & 0.025 & 0.43 & $0.21-0.90$ & 0.064 & 0.45 & $0.20-1.05$ \\
\hline
\end{tabular}




\section{3rd year \\ 4 th year}

Financial contribution to family

Fully dependent on family

Part of earning goes to family

\section{COVID-19 impacted financial situation}

\author{
No impact \\ Yes, impacted positively \\ Yes, impacted negatively
}

Perceived current social life

$$
\text { Dissatisfied }
$$

Satisfied

\section{Smoking}

Never smoker

Ever smoker (Daily/Non-daily/Ex)

Infected with COVID-19

No

Yes

Infected with COVID-19 in the last 14 days

$$
\text { No }
$$

Yes

Experienced symptoms of COVID-19 in the last 14 days

$$
\text { No }
$$$$
\text { Yes }
$$

\section{Contact (indirect/direct) with COVID-19 cases}

$$
\text { No }
$$$$
\text { Yes }
$$

\begin{tabular}{|rr|rr|rrr|rrr|}
63 & 64.3 & 35 & 35.7 & 0.000 & 0.25 & $0.13-0.48$ & 0.000 & 0.17 & $0.08-0.39$ \\
64 & 60.4 & 42 & 39.6 & 0.000 & 0.29 & $0.15-0.56$ & 0.006 & 0.29 & $0.12-0.71$ \\
176 & & 151 & & & & & & & \\
164 & 52.7 & 147 & 47.3 & & 1 & & & 1 & \\
12 & 75 & 4 & 25 & 0.093 & 0.37 & $0.12-1.18$ & 0.225 & 0.45 & $0.12-1.64$ \\
176 & & 151 & & & & & & & \\
49 & 59.8 & 33 & 40.2 & & 1 & & & 1 & \\
31 & 57.4 & 23 & 42.6 & 0.785 & 1.10 & $0.55-2.21$ & 0.764 & 0.88 & $0.38-2.05$ \\
96 & 50.5 & 94 & 49.5 & 0.162 & 1.45 & $0.86-2.46$ & 0.116 & 1.67 & $0.88-3.18$ \\
176 & & 151 & & & & & & & \\
94 & 52.2 & 86 & 47.8 & & 1 & & & 1 & \\
82 & 55.8 & 65 & 44.2 & 0.521 & 0.87 & $0.56-1.34$ & 0.235 & 0.71 & $0.40-1.25$ \\
176 & & 151 & & & & & & & \\
162 & 52.1 & 149 & 47.9 & & 1 & & & 1 & \\
14 & 87.5 & 2 & 12.5 & 0.015 & 0.16 & $0.03-0.69$ & 0.067 & 0.20 & $0.04-1.12$ \\
176 & & 151 & & & & & & & \\
121 & 53.3 & 106 & 46.7 & & 1 & & & 1 & \\
36 & 56.3 & 28 & 43.8 & 0.896 & 0.98 & $0.71-1.35$ & 0.639 & 1.10 & $0.74-1.63$ \\
176 & & 151 & & & & & & & \\
173 & 54.2 & 146 & 45.8 & & 1 & & & 1 & \\
3 & 37.5 & 5 & 62.5 & 0.357 & 1.97 & $0.46-8.40$ & 0.513 & 1.79 & $0.31-10.3$ \\
176 & & 151 & & & & & & & \\
139 & 53.7 & 120 & 46.3 & & 1 & & & 1 & \\
26 & 56.5 & 20 & 43.5 & 0.798 & 0.96 & $0.71-1.30$ & 0.128 & 0.69 & $0.43-1.11$ \\
176 & & 151 & & & & & & & \\
152 & 54.7 & 126 & 45.3 & & 1 & & & 1 & \\
17 & 54.8 & 14 & 45.2 & 0.986 & 0.99 & $0.47-2.09$ & 0.524 & 1.37 & $0.52-3.57$ \\
7 & 38.9 & 11 & 61.1 & 0.199 & 1.90 & $0.71-5.03$ & 0.155 & 2.48 & $0.71-8.71$
\end{tabular}


Experience related to the use of social media

Do not use

Does not affect

Find it irritating

Feel positive about life

Never

Quite often

Always positive

Faced difficulties in adopting distance learning

No

Yes

Level of psychological distress (K10 categories)

Low (score 10-15)

Moderate to Very High (score 16-50)

Level of coping (BRCS categories)

Low resilient copers (score 4-13)

Medium to high resilient copers (score 14-20)

Healthcare service use to overcome COVID-19 related stress in the last 6 months

No

Yes

Type of healthcare service used to overcome COVID-19 related stress in the last 6 months

Consulted a GP

Consulted a Psychologist

Consulted a Psychiatrist

Others

\begin{tabular}{|c|c|c|c|c|c|c|c|c|c|}
\hline 176 & & 151 & & & & & & & \\
\hline 5 & 45.5 & 6 & 54.5 & & 1 & & & 1 & \\
\hline 69 & 63.3 & 40 & 36.7 & 0.254 & 0.48 & $0.14-1.68$ & 0.268 & 0.43 & $0.09-1.93$ \\
\hline 102 & 49.3 & 105 & 50.7 & 0.805 & 0.860 & $0.25-2.90$ & 0.822 & 0.84 & $0.19-3.70$ \\
\hline 176 & & 151 & & & & & & & \\
\hline 12 & 48 & 13 & 52 & & 1 & & & 1 & \\
\hline 93 & 62 & 57 & 38 & 0.190 & 0.57 & $0.24-1.33$ & 0.155 & 0.47 & $0.17-1.33$ \\
\hline 71 & 46.7 & 81 & 53.3 & 0.905 & 1.05 & $0.45-2.46$ & 0.485 & 1.46 & $0.50-4.25$ \\
\hline 176 & & 151 & & & & & & & \\
\hline 26 & 55.3 & 21 & 44.7 & & 1 & & & 1 & \\
\hline 150 & 53.6 & 130 & 46.4 & 0.824 & 1.07 & $0.58-2.00$ & 0.646 & 0.84 & $0.39-1.80$ \\
\hline 176 & & 151 & & & & & & & \\
\hline 26 & 50 & 26 & 50 & & 1 & & & 1 & \\
\hline 150 & 54.5 & 125 & 45.5 & 0.547 & 0.83 & $0.46-1.51$ & 0.190 & 0.58 & $0.26-1.31$ \\
\hline 176 & & 151 & & & & & & & \\
\hline 97 & 49.2 & 100 & 50.8 & & 1 & & & 1 & \\
\hline 79 & 60.8 & 51 & 39.2 & 0.041 & 0.63 & $0.40-0.98$ & 0.140 & 0.67 & $0.39-1.14$ \\
\hline 176 & & 151 & & & & & & & \\
\hline 133 & 54.7 & 110 & 45.3 & & 1 & & & 1 & \\
\hline 43 & 51.2 & 41 & 48.8 & 0.575 & 0.87 & $0.53-1.43$ & 0.934 & 1.03 & $0.55-1.93$ \\
\hline 18 & 51.4 & 17 & 48.6 & 0.501 & 0.73 & $0.29-1.82$ & 0.387 & 2.83 & $0.27-30.0$ \\
\hline 7 & 36.8 & 12 & 63.2 & 0.293 & 1.78 & $0.61-5.19$ & 0.190 & 5.14 & $0.44-59.5$ \\
\hline 7 & 43.8 & 9 & 56.3 & 0.748 & 1.20 & $0.39-3.66$ & 0.284 & 3.86 & $0.33-45.6$ \\
\hline 3 & 75 & 1 & 25 & 0.281 & 0.28 & $0.03-2.83$ & NA & NA & NA \\
\hline
\end{tabular}

Table 7. Predictors for coping among the study population (based on BRCS score) 


\begin{tabular}{|c|c|c|c|c|c|c|c|c|c|c|}
\hline \multirow[t]{2}{*}{ Characteristics } & \multicolumn{2}{|c|}{$\begin{array}{c}\text { Low (score 4- } \\
13)\end{array}$} & \multicolumn{2}{|c|}{$\begin{array}{l}\text { Medium to High } \\
\text { (score 14-20) }\end{array}$} & \multicolumn{3}{|c|}{ Unadjusted analyses } & \multicolumn{3}{|c|}{ Adjusted analyses } \\
\hline & $\mathrm{n}$ & $\%$ & $\mathrm{n}$ & $\%$ & $p$ & ORs & $95 \%$ Cls & $p$ & AORs & $95 \% \mathrm{Cls}$ \\
\hline Age groups & 197 & & 130 & & \multirow{2}{*}{\multicolumn{3}{|c|}{1}} & \multirow{2}{*}{\multicolumn{3}{|c|}{1}} \\
\hline $19-23$ years & 146 & 62.4 & 88 & 37.6 & & & & & & \\
\hline $24-28$ years & 51 & 54.8 & 42 & 45.2 & 0.209 & 1.37 & $0.84-2.22$ & 0.218 & 1.56 & $0.77-3.14$ \\
\hline Gender & 197 & & 130 & & & & & & & \\
\hline Male & 43 & 52.4 & 39 & 47.6 & \multicolumn{3}{|c|}{1} & \multicolumn{3}{|c|}{1} \\
\hline Female & 154 & 62.9 & 91 & 37.1 & 0.096 & 0.65 & $0.39-1.08$ & 0.030 & 0.47 & $0.24-0.93$ \\
\hline Marital status & 197 & & 130 & & & & & & & \\
\hline Married & 26 & 54.2 & 22 & 45.8 & \multicolumn{3}{|c|}{1} & \multicolumn{3}{|c|}{1} \\
\hline Unmarried & 170 & 61.2 & 108 & 38.8 & 0.362 & 0.75 & $0.41-1.39$ & 0.481 & 0.77 & $0.37-1.60$ \\
\hline Family types & 197 & & 130 & & & & & & & \\
\hline Nuclear family & 160 & 60.2 & 106 & 39.8 & \multicolumn{3}{|c|}{1} & \multicolumn{3}{|c|}{1} \\
\hline Joint family & 37 & 60.7 & 24 & 39.3 & 0.942 & 0.98 & $0.55-1.73$ & 0.774 & 0.91 & $0.47-1.76$ \\
\hline Living status & 197 & & 130 & & & & & & & \\
\hline Live without family members & 9 & 81.8 & 2 & 18.2 & \multicolumn{3}{|c|}{1} & \multicolumn{3}{|c|}{1} \\
\hline Live with family members & 82 & 54.3 & 69 & 45.7 & 0.095 & 0.26 & $0.06-1.26$ & 0.051 & 0.16 & $0.03-1.01$ \\
\hline Live in own house & 3 & 42.9 & 4 & 57.1 & 0.556 & 1.58 & $0.34-7.32$ & 0.424 & 2.02 & $0.36-11.4$ \\
\hline Live in shared house & 9 & 56.3 & 7 & 43.8 & 0.882 & 0.92 & $0.33-2.61$ & 0.977 & 1.02 & $0.31-3.33$ \\
\hline Live in hostel & 94 & 66.2 & 48 & 33.8 & 0.038 & 0.61 & $0.38-0.97$ & 0.018 & 0.51 & $0.29-0.89$ \\
\hline Perceived safety of living place in relation to COVID-19 & 197 & & 130 & & & & & & & \\
\hline Unsafe & 27 & 54 & 23 & 46 & \multicolumn{3}{|c|}{1} & \multicolumn{3}{|c|}{1} \\
\hline Safe & 170 & 61.4 & 107 & 38.6 & 0.328 & 0.74 & $0.40-1.36$ & 0.227 & 0.64 & $0.31-1.32$ \\
\hline Year of Dental education & 197 & & 130 & & & & & & & \\
\hline 1st year & 46 & 67.6 & 22 & 32.4 & \multicolumn{3}{|c|}{1} & \multicolumn{3}{|c|}{1} \\
\hline 2nd year & 28 & 50.9 & 27 & 49.1 & 0.061 & 2.02 & $0.97-4.20$ & 0.161 & 1.82 & $0.79-4.20$ \\
\hline 3rd year & 61 & 62.2 & 37 & 37.8 & 0.475 & 1.27 & $0.66-2.43$ & 0.663 & 0.84 & $0.39-1.83$ \\
\hline
\end{tabular}




\section{4th year}

Financial contribution to family

Fully dependent on family

Part of earning goes to family

COVID-19 impacted financial situation

No impact

Yes, impacted positively

Yes, impacted negatively

\section{Perceived current social life}

$$
\text { Dissatisfied }
$$

Satisfied

Smoking

Never smoker

Ever smoker (Daily/Non-daily/Ex)

\section{Infected with COVID-19}

No

$$
\text { Yes }
$$

Infected with COVID-19 in the last 14 days

$$
\text { No }
$$

Yes

Experienced symptoms of COVID-19 in the last 14 days

$$
\text { No }
$$

Yes

\begin{tabular}{|c|c|c|c|c|c|c|c|c|c|}
\hline 62 & 58.5 & 44 & 41.5 & 0.226 & 1.48 & $0.78-2.81$ & 0.493 & 0.74 & $0.31-1.75$ \\
\hline 197 & & 130 & & & & & & & \\
\hline 189 & 60.8 & 122 & 39.2 & & 1 & & & 1 & \\
\hline 8 & 50 & 8 & 50 & 0.394 & 1.55 & $0.57-4.24$ & 0.893 & 1.08 & $0.34-3.49$ \\
\hline 197 & & 130 & & & & & & & \\
\hline 55 & 67.1 & 27 & 32.9 & & 1 & & & 1 & \\
\hline 35 & 64.8 & 19 & 35.2 & 0.785 & 1.11 & $0.54-2.28$ & 0.974 & 1.01 & $0.44-2.34$ \\
\hline 106 & 55.8 & 84 & 44.2 & 0.084 & 1.61 & $0.94-2.78$ & 0.245 & 1.46 & $0.77-2.77$ \\
\hline 197 & & 130 & & & & & & & \\
\hline 104 & 57.8 & 76 & 42.2 & & 1 & & & 1 & \\
\hline 93 & 63.3 & 54 & 36.7 & 0.313 & 0.79 & $0.51-1.24$ & 0.890 & 1.04 & $0.60-1.80$ \\
\hline 197 & & 130 & & & & & & & \\
\hline 190 & 61.1 & 121 & 38.9 & & 1 & & & 1 & \\
\hline 7 & 43.8 & 9 & 56.3 & 0.174 & 2.02 & $0.73-5.56$ & 0.462 & 1.62 & $0.45-5.82$ \\
\hline 197 & & 130 & & & & & & & \\
\hline 143 & 63 & 84 & 37 & & 1 & & & 1 & \\
\hline 35 & 54.7 & 29 & 45.3 & 0.140 & 1.27 & $0.92-1.76$ & 0.093 & 1.39 & $0.95-2.04$ \\
\hline 197 & & 130 & & & & & & & \\
\hline 193 & 60.5 & 126 & 39.5 & & 1 & & & 1 & \\
\hline 4 & 50 & 4 & 50 & 0.552 & 1.53 & $0.38-6.24$ & 0.389 & 2.19 & $0.37-12.9$ \\
\hline 197 & & 130 & & & & & & & \\
\hline 154 & 59.5 & 105 & 40.5 & & 1 & & & 1 & \\
\hline 29 & 63 & 17 & 37 & 0.599 & 0.92 & $0.67-1.26$ & 0.352 & 0.81 & $0.51-1.27$ \\
\hline 197 & & 130 & & & & & & & \\
\hline 164 & 59 & 114 & 41 & & 1 & & & 1 & \\
\hline 23 & 74.2 & 8 & 25.8 & 0.106 & 0.50 & $0.22-1.16$ & 0.096 & 0.42 & $0.15-1.17$ \\
\hline 10 & 55.6 & 8 & 44.4 & 0.774 & 1.15 & $0.44-3.01$ & 0.729 & 1.24 & $0.37-4.12$ \\
\hline 197 & & 130 & & & & & & & \\
\hline
\end{tabular}

Contact (indirect/direct) with COVID-19 cases

$$
\begin{aligned}
& \text { No } \\
& \text { Unsure } \\
& \text { Yes }
\end{aligned}
$$

Experience related to the use of social media 
Do not use

Does not affect

Find it irritating

Feel positive about life

Never

Quite often

Always positive

Faced difficulties in adopting distance learning

No

Yes

Level of psychological distress (K10 categories)

Low (score 10-15)

Moderate to Very High (score 16-50)

Level of fear of COVID-19 (FCV-19S categories)

Low (score 7-21)

High (score 22-35)

Healthcare service use to overcome COVID-19 related stress in the last 6 months

No

Yes

Type of healthcare service used to overcome COVID-19 related stress in the last 6 months Consulted a GP

Consulted a Psychologist

Consulted a Psychiatrist

Others

\begin{tabular}{|c|c|c|c|c|c|c|c|c|}
\hline 63.6 & 4 & 36.4 & \multicolumn{3}{|c|}{1} & \multicolumn{3}{|c|}{1} \\
\hline 65.1 & 38 & 34.9 & 0.921 & 0.94 & $0.26-3.40$ & 0.359 & 0.51 & $0.12-2.14$ \\
\hline \multirow[t]{2}{*}{57.5} & 88 & 42.5 & 0.688 & 1.29 & $0.37-4.56$ & 0.815 & 0.85 & $0.21-3.45$ \\
\hline & 130 & & & & & & & \\
\hline 76 & 6 & 24 & & 1 & & & 1 & \\
\hline 53.3 & 70 & 46.7 & 0.040 & 2.77 & $1.05-7.33$ & 0.026 & 3.67 & $1.17-11.5$ \\
\hline \multirow[t]{2}{*}{64.5} & 54 & 35.5 & 0.264 & 1.74 & $0.66-4.63$ & 0.084 & 2.83 & $0.87-9.20$ \\
\hline & 130 & & & & & & & \\
\hline 72.3 & 13 & 27.7 & & 1 & & & 1 & \\
\hline \multirow[t]{2}{*}{58.2} & 117 & 41.8 & 0.070 & 1.88 & $0.95-3.71$ & 0.120 & 1.87 & $0.85-4.09$ \\
\hline & 130 & & & & & & & \\
\hline 65.4 & 18 & 34.6 & & 1 & & & 1 & \\
\hline \multirow[t]{2}{*}{59.3} & 112 & 40.7 & 0.410 & 1.30 & $0.70-2.41$ & 0.890 & 0.94 & $0.42-2.11$ \\
\hline & 130 & & & & & & & \\
\hline 55.1 & 79 & 44.9 & & 1 & & & 1 & \\
\hline \multirow[t]{2}{*}{66.2} & 51 & 33.8 & 0.041 & 0.63 & $0.40-0.98$ & 0.131 & 0.66 & $0.38-1.13$ \\
\hline & 130 & & & & & & & \\
\hline 56.4 & 106 & 43.6 & & 1 & & & 1 & \\
\hline 71.4 & 24 & 28.6 & 0.016 & 1.93 & $1.13-3.31$ & 0.017 & 2.19 & $1.15-4.17$ \\
\hline 68.6 & 11 & 31.4 & 0.421 & 1.53 & $0.54-4.29$ & 0.793 & 1.38 & $0.13-14.8$ \\
\hline 84.2 & 3 & 15.8 & 0.210 & 0.42 & $0.11-1.63$ & 0.662 & 0.56 & $0.04-7.40$ \\
\hline 68.8 & 5 & 31.3 & 0.668 & 1.30 & $0.39-4.38$ & 0.808 & 1.36 & $0.11-16.6$ \\
\hline 75 & 1 & 25 & 0.925 & 0.89 & $0.09-9.14$ & NA & NA & NA \\
\hline
\end{tabular}




\section{Discussion}

This is one of the very few studies conducted in Bangladesh amongst dental students about their mental health impacts during the current COVID-19 pandemic. Levels of psychological distress, fear and attempt to overcome the impact of ongoing pandemic was assessed; factors associated with those issues were also identified. Medical and dental education are considered as highly stressful globally, because medical or dental students experience high level of anxiety, stress and depression in comparison to students studying in other subjects [30-32]. After emergence of the COVID-19 pandemic, dental education had been affected significantly owing to the need for reducing face to face contact and enforcing social distancing in communities. Although several studies were conducted regarding psychological impact and fear of COVID-19 among general population and medical students, this cross-sectional study was the first ever carried out in Bangladesh among dental students to assess the severity and identify factors associated with psychological distress, levels of fear and coping strategies during the COVID-19 pandemic.

In this study, most of the dental students had moderate to high level of psychological distress (84.1\%). That level was significantly higher than the medical students (65.9\%) [33], general students (18.1\%) [34] and general population (30.1\%) [35]. In Bangladesh during the COVID-19 pandemic. This might be due to increased risk of exposure of COVID-19 among dental students. Moreover, prior evidence indicated that dental education used to generate more stress and burnout than medical educations [36]. Due to more interactive involvement with patient during theoretical and clinical courses [37]. In this study, 3rd year clinical students were more prone to have psychological distress due to COVID-19. Similar finding was reported in other studies, where clinical years had moderate to high levels of stress [38]. Another study by AL-Sowygh et.al showed that 3rd year students had more stress due to performance pressure during clinical examination [22,39]. In this study those who were infected with COVID-19 and who were unsure about the direct or indirect contact of COVID-19 cases were more prone to develop psychological distress. Similar finding was reported in the study conducted among Australian population [6]. Those respondents in this study who reported negative impact on their financial situations tended to have moderate to very high levels of psychological distress. As most of the dental students who took part in this study were fully dependent on their families, the negative financial impact could have hampered their academic progress.

Low levels of fear were reported amongst dental students in this study. That finding was in contrast to the finding from another study conducted in Bangladesh, which reported higher levels of fear amongst frontline or essential service workers [5]. Nevertheless, study finding from this study was consistent with the findings of another study where COVID-19 related fear was low among frontline health care workers. Similarly, low levels of fear among the doctors was observed in another study [6]. This might be due to increased engagement with the patients with a higher risk of exposure of COVID-19 and the availability of the protective gear during the time of data collection in Bangladesh. In this study, female dental students had higher levels of fear. Similar trends were observed among female dental and medical students and general population conducted elsewhere in Bangladesh [33,6,40,41,42]. This might be due to their inherent caregivers' roles both in profession and families, hormonal changes, and expression of emotions, which could have contributed to the increased intensity of fear of COVID-19. In this study, 3rd and 4th year clinical dental students had low levels of fear which was similar to other studies conducted among doctors [7]. Medium to high resilient copers were more likely to have low level of fears in this study, which could be explained by the inherent capacity of high resilient copers to manage their fear, emotion, and stress more positively than the low resilient copers. Study participants who used healthcare services to combat COVID-19 related stress were more likely to be medium to high resilient copers. Similar findings were also reported in earlier study, where visiting healthcare providers in persons was associated with high level of coping during the COVID-19 pandemic [7]. 
On the other hand, female students and students living in hostel tended to be low resilient copers. Students who had been living in hostels could have been dealing with a variety of concerns such as financial difficulties, home sickness, concerns on safety of parents and relatives, change in sleeping and eating habit, and issues adjusting to their new surroundings, all of which probably made them more susceptible to psychological distress, hence low coping. Overall, this study identified that study participants were more low resilient copers, which could be due to high female respondents in this study. So, in order to properly analyze these concerns, further research and study need to be conducted.

This study had few limitations. It was conducted among the students of two private dental colleges situated in Dhaka, Bangladesh, hence findings could not be generalized for all the dental students of Bangladesh. This was an online based study, therefore the students who were only active in online and had better internet connection were more likely to respond in this study. In addition, distressed students were more likely to respond in this study which might have resulted in selection bias. However, considering the ongoing pandemic crisis, it was inevitable to collect data online because of restriction of movement and social distancing. Nevertheless, this study was first of its kind in Bangladesh to reveal the psychological distress, fear, and coping strategies of dental undergraduate students in Bangladesh.

\section{Conclusions}

This study identified that most of the dental students experienced moderate to very high levels of psychological distress while half of them had low levels of fear of COVID-19 with most of them being low resilient copers. The factors identified in this study should be considered in addressing mental health impacts of dental students in Bangladesh. Developing policies and support strategies for addressing health and wellbeing of dental students is imperative besides the core support for academic and clinical skills development. Future studies could focus on stakeholders and students of both public and private dental institutions in Bangladesh about the specific support strategies for psychological wellbeing during and post-pandemic era.

\section{Supplementary Materials: Nil}

Author Contributions: Conceptualization, F.S. and M.A.R.; methodology, M.A.R.; formal analysis, M.A.R.; investigation, F.S., M.T.H.C., S.K.N., A.A.I., S.M.A.Q., M.S.J., A.E.N., C.P.P., U.G., R.N.; writing-original draft preparation, F.S., M.T.H.C., M.A.R.; supervision, M.A.R. All authors have read and agreed to the published version of the manuscript.

Funding: The research was not funded.

Institutional Review Board Statement: The study was conducted according to the guidelines of the Declaration of Helsinki and approved by the Institutional Review Board (or Ethics Committee) of Sapporo Dental College \& Hospital (Ref: SDC/C-7/2021/829; Date of approval: 27-Oct-2021).

Informed Consent Statement: Informed consent was obtained from all subjects involved in the study. Any information which could identify any individual were not collected.

Data Availability Statement: The data are available upon reasonable request from the corresponding author.

Acknowledgments: We would like to appreciate the support and participation of all the students who participated in this study.

Conflicts of Interest: The authors declare no conflicts of interest.

1. Worldometer. COVID-19 Coronavirus Pandemic. (2021). Available online at: https:// www. worldometers.info/coronavirus/ (accessed 29 November, 2021). 
2. Directorate General of Health Services (DGHS) Bangladesh. Coronavirus COVID 19 Dashboard. (2021). Available online at: http://103.247.238.92/ webportal/pages/covid19.php (accessed 29 November 2021)].

3. World Health Organization Bangladesh. COVID-19 2020. Available online at: https:// www.who.int/docs/default-source/searo/bangladesh/covid-19-who-bangladesh-situation-reports/who_covid19update_39_20201123.pdf?sfvrsn=ebe679c6_11 (accessed November 29 2021).

4. Zubayer AA, Rahman ME, Islam, MB, Babu SZD, Rahman QM, et al. Psychological states of Bangladeshi people four months after the COVID-19 pandemic: an online survey. Heliyon. (2020) 6:e05057. doi: 10.1016/j.heliyon.2020.e05057.

5. Rahman MA, Rahman S, Wazib A, Arafat SMY, Chowdhury ZZ, Uddin BMM, Rahman MM, Bahar Moni AS, Alif SM, Sultana F, Salehin M, Islam SMS, Cross W and Bahar T COVID-19 Related Psychological Distress, Fear and Coping: Identification of High-Risk Groups in Bangladesh (2021) Front.Psychiatry12:718654. doi:10.3389/fpsyt.2021.718654

6. Rahman MA, Hoque N, Alif SM, Salehin M, Islam SMS, Banik B, Sharif A, Nazim NB, Sultana F, Cross W. Factors associated with psychological distress, fear and coping strategies during the COVID-19 pandemic in Australia. Global Health. 2020 Oct 8;16(1):95. doi: 10.1186/s12992-020-00624-w. PMID: 33032629; PMCID: PMC7542573.

7. Rahman MA, Islam SMS, Tungpunkom P, Sultana F, Alif SM, Banik B, Salehin M, Joseph B, Lam L, Watts MC, Khan SJ, Ghozy S, Chair SY, Chien WT, Schönfeldt-Lecuona C, El-Khazragy N, Mahmud I, Al Mawali AH, Al Maskari TS, Alharbi RJ, Hamza A, Keblawi MA, Hammoud M, Elaidy AM, Susanto AD, Bahar Moni AS, AlQurashi AA, Ali A, Wazib A, Sanluang CS, Elsori DH, Yasmin F, Taufik FF, Al Kloub M, Ruiz MG, Elsayed M, Eltewacy NK, Al Laham N, Oli N, Abdelnaby R, Dweik R, Thongyu R, Almustanyir S, Rahman S, Nitayawan S, Al-Madhoun S, Inthong S, Alharbi TA, Bahar T, Ginting TT, Cross WM. COVID-19: Factors associated with psychological distress, fear, and coping strategies among community members across 17 countries. Global Health. 2021 Oct 1;17(1):117. doi: 10.1186/s12992-021-00768-3. PMID: 34598720; PMCID: PMC8485312.

8. Qiu, J., Shen, B., Zhao, M., Wang, Z., Xie, B., Xu, Y., 2020. A nationwide survey of psychological distress among Chinese people in the COVID-19 epidemic: Implications and policy recommendations. General Psychiatry 33, e100213. doi:10.1136/gpsych-2020-100213.

9. Banerjee, D., 2020. The COVID-19 outbreak: Crucial role the psychiatrists can play. Asian Journal of Psychiatry 50, 102014. doi:10.1016/j.ajp.2020.102014.

10. Kabir, R.; Isha, S.N.;Chowdhury, M.T.H.; Siddika, N.;Jahan, S.S.; Saha, A.K.; Nath, S.K.;Jahan, M.S.; Sivasubramanian, M.;Mahmud, I.; et al. Depression among the Non-Native International Undergraduate Students Studying Dentistry in Bangladesh. Int. J. Environ. Res. Public Health 2021, 18, 5802. https://doi.org/10.3390/ijerph18115802

11. Bathla, M.; Singh, M.; Kulhara, P.; Chandna, S.; Aneja, J. Evaluation of anxiety, depression and suicidal intent in under-graduate dental students: A cross-sectional study. Contemp. Clin. Dent. 2015, 6, 215-222.

12. Deeb, G.R.; Braun, S.; Carrico, C.; Kinser, P.; Laskin, D.; Deeb, J.G. Burnout, depression and suicidal ideation in dental and den-tal hygiene students. Eur. J. Dent. Educ. 2017, 22, e70-e74.

13. Meisha et al. Social determinants of seeking emergency and routine dental care in Saudi Arabia during the COVID-19 pan-demic BMC Oral Health (2021) 21:212 https://doi.org/10.1186/s12903-021-01577-1.

14. Sarwar H. A Nation-wide Survey on Financial Impact of COVID-19 on Employers of Private Dental Practices of Pakistan JPDA 2020; 29: 172-8.

108 
15. Coulthard P. Dentistry and coronavirus (COVID-19)- moral decision-making. Br Dent J 2020 ;228: 503-5.

16. Majeed MM, Siddiqui Z, Tabbasum U, Sarwar H, Minhas S, Bhatia MR Psychological barriers among dental patients during the COVID-19 crisis, Rawal Medical Journal: Vol. 46. No. 1, Jan.-Mar. 2021.

17. Majeed MM, Durrani MS, Bashir MB, Ahmed M. COVID-19 and Dental Education in Pakistan. J Coll Physicians Surg Pak JCPSP 2020; 30: 115-7.

18. Jiang CM, Duangthip D, Auychai P, Chiba M, Folayan MO, Hamama HHH, Kamnoedboon P, Lyons K, Matangkasombut O, Ma-thu-Muju KR, Mathur VP, Mei ML, Morgan M, Poolthong S, Rahul M, Srinivasan M, Takahashi T, Yaklai S, Zhang S, Zou XC, Chu CH and Lo ECM (2021) Changes in Oral Health Policies and Guidelines During the COVID-19 Pandemic. Front. Oral. Health 2: 668444. doi:10.3389/froh.2021.668444.

19. Barabari P, Moharamzadeh K. Novel Coronavirus (COVID-19) and Dentistry-A Comprehensive Review of Literature. Dent J (Basel). 2020 May 21;8(2):53. doi: 10.3390/dj8020053. PMID: 32455612; PMCID: PMC7345990.

20. Emami E. COVID-19: Perspective of a Dean of Dentistry. JDR Clin Trans Res. 2020 Jul;5(3):211-213. doi: 10.1177/2380084420929284. Epub 2020 May 13. PMID: 32401587.

21. Iyer P, Aziz K, Ojcius DM. Impact of COVID-19 on dental education in the United States. J Dent Educ. 2020; In Press

22. Zaki Hakami ,Sanjeev B. Khanagar ,Satish Vishwanathaiah,Abrar Hakami,Ahmed M. Bokhari,Ahmad H. Jabali, Dhafer Al asmari,Abdullah M. Aldrees Psychological impact of the coronavirus disease 2019(COVID19) pandemic on dental students: A nationwide study J Dent Educ. 2021; 85: 494-503. doi: 10.1002/jdd.12470

23. Lloyd C, Musser LA. Psychological impact of the coronavirus disease 2019 (COVID-19) pandemic on dental students: A nation-wide study psychiatric symptoms in dental students. JNervMent Dis. 1989; 177: 61-69.

24. HumphrisG, BlinkhornA, Freeman R, et al. Psychological stress in undergraduate dental students: baseline results from seven European dental schools. Eur J Dent Educ. 2002; 6(1): 22-29.

25. Uraz A, Tocak YS, Yozgatligil C, Cetiner S, Bal B. Psychological well-being, health, and stress sources in Turkish dental students. J Dent Educ. 2013; 77(10): 1345-1355.

26. Montero-Marin J, Piva DMM,Stapinski L, Gili M, Garcia- Campayo J. Perceived stress latent factors and the burnout subtypes: a structural model in dental students. PLoS One.2014; 9(6): e99765.

27. Furukawa TA, Kessler RC, Slade T. Andrews G. The performance of the K6 and K10 screening scales for psychological distress in the Australian National Survey of Mental Health and Well-Being. Psychol Med. (2003) 33: 357-62. doi: 10.1017/S0033291702006700

28. AhorsuDK, Lin CY, Imani V, SaffariM, GriffithsMD, Pakpour AH. The fear of COVID-19 scale: development and initial validation. Int JMent Health Addict. (2020). doi: 10.1007/s11469-020-00270-8. [Epub ahead of print].

29. Sinclair VG, Wallston KA. The development and psychometric evaluation of the Brief Resilient Coping Scale. Assess-ment. (2004) 11: 94-101. doi: 10.1177/1073191103258144

30. Aboalshamat K, Hou X-Y, Strodl E. Psychological well-being status among medical and dental students in Makkah, Sau-di Arabia: a cross-sectional study. Med Teach. 2015; 37 Suppl 1(sup1): S75-81.

31. Eva EO, Islam MZ, Mosaddek ASM, Rahman MF, Rozario RJ, Iftekhar AFMH, et al. Prevalence of stress among medical stu-dents: a comparative study between public and private medical schools in Bangladesh. BMC Res Notes [Internet]. 2015 ;8(1). Available from: http://dx.doi.org/10.1186/s13104-015-1295-5

32. Hasan MT, Hossain S, Gupta RD, Podder V, Mowri NA, Ghosh A, et al. Depression, sleeping pattern, and suicidal idea-tion among medical students in Bangladesh: a cross-sectional pilot study. Z Gesundh Wiss [Internet]. 2020; Available from: http://dx.doi.org/10.1007/s10389-020-01304-0 
33. Safa F, Anjum A, Hossain S, Trisa TI, Alam SF, Abdur Rafi M, et al. Immediate psychological responses during the initial period of the COVID-19 pandemic among Bangladeshi medical students. Child Youth Serv Rev. 2021; 122(105912): 105912.

34. Far Abid Hossain S, Nurunnabi M, Sundarasen S, Chinna K, Kamaludin K, Baloch GM, et al. Socio-psychological impact on Bang-ladeshi students during COVID-19. J Public Health Res. 2020; 9(Suppl 1): 1911.

190 191

38. Naidu RS, Adams JS, Simeon D, Persad S. Sources of stress and psychological disturbance among dental students in the West In-dies. J Dent Educ. 2002; 66(9): 1021-30

39. Al-Sowygh ZH, Alfadley AA, Al-Saif MI, Al-Wadei SH. Perceived causes of stress among Saudi dental students. King Saud Univ j dent sci. 2013; 4(1): 7-15.

40. Gausman J, Langer A. Sex and Gender Disparities in the COVID-19 Pandemic. J Womens Health (Larchmt). 2020; 29(4): 4656

41. Pappa S, Ntella V, Giannakas T, Giannakoulis VG, Papoutsi E, Katsaounou P. Prevalence of depression, anxiety, and in-som-nia among healthcare workers during the COVID-19 pandemic: A systematic review and metaanalysis. Brain Behav Immun. 2020; 88: 901-7. 